\title{
ATENÇÃO À SAÚDE DO RECÉM-NASCIDO E DA CRIANÇA INDÍGENA: PERSPECTIVAS DE ATUAÇÃO DO ENFERMEIRO
}

\author{
HEALTH CARE OF NEWBORN AND INDIGENOUS CHILDREN: \\ PERSPECTIVES OF NURSING PRACTICE
}

\section{ATENCIÓN DE SALUD DEL RECIÉN NACIDO Y NIÑOS INDÍGENAS: PERSPECTIVAS DE LA PRÁCTICA DE ENFERMERÍA}

\author{
João Paulo Assunção Borges ${ }^{1}$, Sarah Mendes de Oliveira ${ }^{2}$
}

\begin{abstract}
RESUMO
Objetivos: Identificar as ações de Atenção à Saúde do recém-nascido $(\mathrm{RN})$ e criança indígena, apontando práticas de cuidado em interface com a atuação do enfermeiro. Método: Trata-se de Revisão Integrativa, composta por artigos obtidos nas bases de dados científicos, utilizando-se os descritores: recém-nascido; criança; indígena; enfermagem. Os artigos foram organizados por meio de formulário para coleta de dados. Resultados: Foram encontrados 09 artigos publicados entre 2006-2013. A primeira categoria abordou fatores associados a déficits nutricionais, como Baixo Peso (BP) para idade, déficits estaturais e BP ao nascer. A segunda categoria retratou cuidados com diarreia e infecções. A terceira categoria apresentou a compreensão popular e da enfermagem sobre morte de crianças indígenas. A quarta categoria identificou práticas de cuidado com RN indígena. Conclusão: O enfermeiro deve conhecer e compreender os aspectos do processo de cuidado dos $\mathrm{RN}$ e crianças indígenas, aprimorando suas estratégias de atuação frente a este grupo.
\end{abstract}

Descritores: Recém-nascido; Criança; População Indígena; Enfermagem.

\begin{abstract}
Objectives: To identify the actions to Health Care of the newborn (NB) and indigenous child care practices in pointing interface with the work of nurses. Method: This is an integrative literature review, made up articles obtained in scientific databases, using the key-words: newborn; child; indigenous; nursing. The articles were organized through a form for collecting data. Results: We found 09 articles published between 2006-2013. The first category addressed factors associated with nutritional deficiencies such as low weight (LW) for age, stature deficits and LW at birth. The second category portrayed care diarrhea and infections. The third category presented the popular understanding and nurses on death of indigenous children. The fourth category identified care practices with indigenous RN. Conclusion: The nurse should know and understand aspects of the care process of NB and indigenous children, improving their operational strategies in front of this group.

Descriptors: Newborn; Children; Indigenous Population; Nursing.

\footnotetext{
${ }^{1}$ Enfermeiro do Hospital de Clínicas da UFU. Doutorando em Atenção à Saúde. Universidade Federal do Triângulo Mineiro. E-mail: enf_joaopaulo@yahoo.com.br.

${ }^{2}$ Acadêmica do Curso de Graduação em Enfermagem. Universidade Federal de Uberlândia (UFU). Av. Pará, 1720. Bairro

Umuarama, Uberlândia, Minas Gerais. E-mail: sarahmendes.ufu@gmail.com.
} 


\section{RESUMEN}

Objetivos: Identificar las acciones y Cuidados de la Salud del recién nacido (RN) y los niños indígenas, señalando las prácticas de atención interconectados con el trabajo de los enfermeros. Método: Se trata de revisión integradora, formada por los artículos obtenidos en las bases de datos científicos, utilizando las palabras-clave: recién nacido; niño; indígena; enfermería. Los artículos se organizáron por meio de un formulario de recogida de datos. Resultados: Se encontraron 09 artículos publicados entre 2006-2013. La primera categoría se dirigió a los factores asociados a deficiencias nutricionales, como bajo peso (BP) para la edad, estaturais déficit y BP en el nacimiento. La segunda categoría retratado diarrea cuidado y las infecciones. La tercera categoría presentó la comprensión popular y enfermeras sobre la muerte de los niños indígenas. La cuarta categoría identificó las prácticas de cuidado con RN indígena. Conclusión: Lo enfermero debe conocer y entender los aspectos del proceso de atención de los $\mathrm{RN}$ y los niños indígenas, mejorando sus estrategias operacionales frente a este grupo.

Descriptores: Recién nacido; Niño; Población indígena; Enfermería.

\section{INTRODUÇÃO}

A atenção à saúde da criança indígena tem como prioridades as estratégias de promoção, proteção e prevenção de agravos por meio da implementação de políticas e programas visando à melhoria das condições socioambientais, culturais e de saúde, considerando as suas especificidades culturais. A assistência e a promoção da saúde para a população indígena podem melhorar as condições de saúde e qualidade de vida dessa população. ${ }^{1,2,3}$

Diante da situação atual do componente de mortalidade infantil brasileira, sobretudo no que se refere à mortalidade neonatal, são necessárias intervenções com vistas à modificação dos determinantes das condições de saúde e doença dos Recém-Nascidos (RN) e crianças. Incidência elevada de Doenças Infecto-Contagiosas (DIC), distúrbios nutricionais e déficit de crescimento e desenvolvimento são problemas frequentes na população neonatal, inclusive entre os RN indígenas. Nesse contexto, as ações de Atenção à Saúde do RN e criança indígena devem ser planejadas e implementadas por gestores e profissionais da saúde com a participação das comunidades envolvidas, preservando e respeitando os aspectos sócio-culturais da população indígena. ${ }^{1,2,3,4}$

A Enfermagem representa uma das profissões da área da saúde com grande inserção nos diversos contextos da sociedade, inclusive nas comunidades indígenas. Desenvolve ações diversificadas, tais como atividades assistenciais individuais e coletivas, educação em saúde da comunidade, 
capacitação e educação permanente de profissionais de Enfermagem, gestão de unidades, serviços e programas de saúde, planejamento e implementação de ações e políticas de saúde, assessoria, consultoria e auditoria, além de atividades de pesquisa e produção científica. Definem, ainda, a atuação do enfermeiro as atividades de promoção, prevenção, recuperação, reabilitação à saúde e trabalho em equipe. ${ }^{5}$ Ressalta-se que o enfermeiro é um dos profissionais de saúde que tem como função a assistência imediata e o acompanhamento do crescimento e desenvolvimento do $\mathrm{RN}$ e criança, inclusive indígena.

Algumas dificuldades são enfrentadas pela população indígena no acesso aos serviços de saúde, como a falta de conhecimento e/ou percepções negativas dos profissionais de saúde relacionadas aos sistemas culturais e aos seus praticantes. ${ }^{6}$ Diante disso, é importante que os profissionais atentem para importância do respeito aos povos indígenas, abordando-os de forma holística. Neste sentido objetivou-se identificar as ações de Atenção à Saúde do $\mathrm{RN}$ e criança indígena, apontando práticas de cuidado em interface com a atuação do enfermeiro.

\section{MÉTODO}

Na busca por conhecimento sobre o cuidado ao RN e criança indígena, as práticas adotadas nas comunidades, assim como o papel do enfermeiro nestas práticas, optou-se pelo método da Revisão Integrativa (RI). Esta metodologia de pesquisa permite a inclusão de literatura teórica e empírica, bem como estudos com diferentes abordagens metodológicas, facilitando o acesso a evidências científicas recentes e favorecendo o aprimoramento do conhecimento acerca de determinado assunto, além de evidenciar pontos que requerem maior enfoque de novas pesquisas. ${ }^{5,7}$

Neste processo de RI, foram realizadas as seguintes etapas: (1) escolha e delimitação do tema, (2) pesquisa nas bases de dados, (3) coleta de dados e categorização, (4) análise e avaliação dos artigos incluídos na revisão, interpretação e discussão dos resultados, (6) síntese e apresentação dos resultados de forma descritiva. ${ }^{5}$ Para análise dos artigos utilizou-se um instrumento não validado, denominado Formulário para Coleta de Dados em Pesquisa Bibliográfica (Anexo A), elaborado por Moura-Ferreira ${ }^{8}$, composto pelos seguintes itens: dados 
referentes ao artigo ou periódico; dados referentes aos pesquisadores; dados referentes ao estudo/pesquisa; resultados; conclusões e considerações finais.

Foram consultadas as bases de dados científicas Medline (Literatura Internacional) e Literatura Latino Americana do Caribe (Lilacs), sendo selecionados os artigos publicados entre janeiro de 2004 e janeiro de 2014, na área de Saúde Coletiva, Saúde da População Indígena, Pediatria, Neonatologia, Enfermagem Pediátrica e Neonatal. Para assegurar uma busca criteriosa dos artigos, foram delimitados os seguintes descritores controlados em Ciências da Saúde: recémnascido, criança, indígena, enfermagem. Foi realizada a leitura dos títulos e resumos dos trabalhos encontrados, disponíveis on line, na íntegra.
Os critérios de inclusão foram: artigos completos, disponíveis on line na íntegra, escritos na língua portuguesa, publicados entre 2004 e 2014. Adotou-se como critério de exclusão: artigos publicados fora do período determinado e cujo tema não se refere ao tema central desta pesquisa.

\section{RESULTADOS E DISCUSSÃO}

Foram obtidos 9 artigos na composição da amostra desta RI, após aplicação dos critérios de inclusão e exclusão. $\mathrm{Na}$ etapa de coleta de dados e categorização dos estudos foi feita a leitura minuciosa dos artigos completos que foram selecionados.

Tabela 1: Tabela de apresentação dos artigos da amostra da RI. UberlândiaMG, 2015.

\begin{tabular}{|c|c|c|c|}
\hline Autores & Nome do Artigo & $\begin{array}{l}\text { Ano de } \\
\text { publicação }\end{array}$ & Objetivo (s) \\
\hline $\begin{array}{l}\text { OLIVEIRA, P.R; } \\
\text { SHIRMBECK, } \\
\text { T.M; LUNARDI, } \\
\text { R.R. }\end{array}$ & $\begin{array}{l}\text { Vivências de uma equipe de } \\
\text { Enfermagem com a morte de } \\
\text { criança indígena hospitalizada. } \\
\text { (A1) }\end{array}$ & 2013 & $\begin{array}{l}\text { Conhecer as experiências da equipe de } \\
\text { enfermagem, com a morte de uma criança } \\
\text { indígena hospitalizada e os sentimentos que } \\
\text { emergiram dessa vivência. }\end{array}$ \\
\hline $\begin{array}{l}\text { RISSARDO, L.K; } \\
\text { MOLITERNO, } \\
\text { A.C.M; BORGHI, } \\
\text { A.C; } \\
\text { CARREIRA, L. }\end{array}$ & $\begin{array}{l}\text { Práticas de Cuidado ao } \\
\text { Recém-Nascido: Percepção de } \\
\text { Famílias Kaingang. (A2) }\end{array}$ & 2011 & $\begin{array}{l}\text { Descrever e analisar as práticas de cuidado ao } \\
\text { recém-nascido a partir da percepção de } \\
\text { mulheres indígenas. }\end{array}$ \\
\hline $\begin{array}{l}\text { BASTA, P.C; } \\
\text { RIOS, D.P.G; } \\
\text { ALVES, L.C.C; }\end{array}$ & $\begin{array}{l}\text { Estudo clínico-radiológico de } \\
\text { crianças e adolescentes } \\
\text { indígenas Suruí, Região }\end{array}$ & 2010 & $\begin{array}{l}\text { Descrever as características clínico- } \\
\text { radiológicas em crianças e adolescentes com } \\
\text { diagnóstico de Tuberculose. Além disto, }\end{array}$ \\
\hline
\end{tabular}


SANT'ANNA, C.C; COIMBRA JUNIOR, C.E.A.

Amazônica. (A3)

FERREIRA, A.A. Estado Nutricional e Fatores Associados ao Crescimento de Crianças Indígenas Xavante, Mato Grosso. (A4)

PÍCOLI, R.P; $\quad$ Cuidado à Saúde de Crianças ADORNO, R.C.F Kaiowá e Guarani: notas de observação de campo. (A5)

PÍCOLI, R.P. Saúde, Doença E Morte De Crianças: Um Olhar Segundo A Percepção Dos Kaiowá E Guarani. (A6)

$\begin{array}{llrr}\text { LEITE, M.S; } & \text { Sazonalidade e estado } \\ \text { SANTOS, R.V; } & \text { nutricional de populações } \\ \text { COIMBRA } & \text { indígenas: o caso Wari, } \\ \text { JUNIOR, C.E.A. } & \text { Rondônia, Brasil. (A7) }\end{array}$

MONDINI, L.; $\quad$ Condições de nutrição em CANÓ, E.N.; crianças Kamaiurá - povo FAGUNDES, U.; indígena do Alto Xingu, Brasil LIMA, E.E.S.; $\quad$ Central. (A8) RODRIGUES D.;

BARUZZI, R.G.

MENEGOLLA, Estado nutricional e fatores I.A; associados à estatura de DRACHLER, crianças da Terra Indígena M.L;

RODRIGUES

I.H.;

SCHWINGEL,

L.R;

SCAPINELLO,

E. PEDROSO,

M.B; LEITE,

J.C.C. aplicar o sistema de pontuação para o diagnóstico de Tuberculose na infância e verificar se as condutas adotadas no nível local foram concordantes com as diretrizes nacionais.

2009 Descrever o estado nutricional e analisar os fatores associados ao crescimento de 232 crianças indígenas Xavante de Pimentel Barbosa/Etênhiritipá (MT).

2008 Identificar as práticas de saúde infantil em diferentes idades do ciclo de vida da pessoa Kaiowá e Guarani, principalmente relacionadas à diarreia infantil.

2008 Investiga o significado de saúde e doença infantil, propondo identificar as explicações e as circunstâncias em que ocorreu o óbito de crianças menores de um ano de vida, segundo a percepção desse povo.

2007 Descrever a antropometria, com atenção às flutuações sazonais das condições de nutrição, à identificação dos grupos populacionais afetados.

2007 Avaliar o estado nutricional e a concentração de hemoglobina sérica de crianças Kamaiurá, povo indígena do Alto Xingu, no Brasil Central.

2006 Descrever o estado nutricional das crianças menores de cinco anos e estudar determinantes sociais do crescimento linear, indicador das condições de vida e saúde da população.

Fonte: Os autores. 
A análise dos artigos permitiu Práticas de cuidado com o $\mathrm{RN}$ indígena compor as seguintes categorias: (1) (Tabela 2).

Avaliação Nutricional (relação peso/altura, altura/idade, peso/idade); (2) Principais

Tabela 2: Frequência e porcentagem distúrbios na criança indígena: diarreia, desnutrição e infecções; (3) Percepção da das categoriais obtidas nos artigos da RI. morte de crianças indígenas, e por fim, (4)

Uberlândia-MG, 2015.

\begin{tabular}{|c|c|c|c|c|}
\hline & & Categorias & & \\
\hline & $\begin{array}{l}\text { Avaliação } \\
\text { nutricional }\end{array}$ & $\begin{array}{l}\text { Principais distúrbios } \\
\text { na criança indígena }\end{array}$ & $\begin{array}{l}\text { Percepção da morte } \\
\text { de criança indígena }\end{array}$ & $\begin{array}{l}\text { Práticas de cuidado } \\
\text { com o RN indígena }\end{array}$ \\
\hline Frequência (n) & 4 & 2 & 2 & 1 \\
\hline Porcentagem (\%) & $44 \%$ & $22 \%$ & $22 \%$ & $11 \%$ \\
\hline Autores & $\begin{array}{c}\text { A4; A7; A8; } \\
\text { A9. }\end{array}$ & A3; A5. & A1; A6. & $\mathrm{A} 2$. \\
\hline $\begin{array}{ll}\text { Período } & \text { de } \\
\text { Publicação } & \end{array}$ & $2006-2009$ & $2008-2010$ & $2008-2013$ & 2011 \\
\hline
\end{tabular}

Fonte: Os autores.

A primeira categoria encontrada em necessário fazer o uso dos índices 44\% (04) da amostra de artigos foi peso/idade (P/I), peso/altura (P/A) e denominada “Avaliação Nutricional”. O altura/idade (A/I), cujos valores, a partir estado nutricional de uma população está das recomendações da OMS, devem ser diretamente relacionado com sua qualidade comparados com a população de referência de vida. Grupos populacionais considerados vulneráveis e determinadas faixas etárias, como as crianças menores de cinco anos, estão entre as mais atingidas por distúrbios nutricionais, em particular, pelas carências nutricionais. ${ }^{9}$ Para a avaliação nutricional de crianças, é do National Center of Health Statistics NCHS. $^{3}$

Os fatores determinantes no déficit de crescimento e na anemia baseiam-se nas condições inadequadas e insatisfatórias de higiene, alimentação e habitação e dificuldade de acesso aos serviços de saúde

\footnotetext{
${ }^{1}$ Enfermeiro do Hospital de Clínicas da UFU. Doutorando em Atenção à Saúde. Universidade Federal do Triângulo Mineiro. E-mail: enf_joaopaulo@yahoo.com.br.

${ }^{2}$ Acadêmica do Curso de Graduação em Enfermagem. Universidade Federal de Uberlândia (UFU). Av. Pará, 1720. Bairro Umuarama, Uberlândia, Minas Gerais. E-mail: sarahmendes.ufu@gmail.com.
} 
às quais as crianças indígenas podem estar submetidas. A baixa estatura de crianças indígenas está relacionada diretamente com a baixa idade e escolaridade materna, porque essas mães tendem a ter menor habilidade e menor comprometimento com o cuidado da criança. ${ }^{10} \mathrm{O}$ baixo peso ao nascer e baixo peso para idade, apontados em menor ocorrência, porém, tendo importância para a saúde de crianças indígenas, foram evidenciados pelas dificuldades socioeconômicas das famílias indígenas, dificuldade de acesso aos serviços de saúde, baixa cobertura de assistência pré-natal e principalmente, o desmame precoce. ${ }^{11,12}$

O enfermeiro é o principal educador em saúde durante o pré-natal e no puerpério. No pré-natal assume grande importância, podendo dialogar com a gestante indígena sobre as vantagens de uma amamentação plena. No puerpério, é o profissional mais capacitado para habilitar a puérpera a respeito dos exercícios facilitadores da ejeção do leite, da alimentação mais adequada durante o aleitamento e da adesão de fatores ambientais favoráveis à efetuação de uma experiência aprazível e saudável durante a amamentação, evitando assim, déficits nutricionais. Lembrando sempre do respeito à cultura da população.

A segunda categoria encontrada em $22 \%$ (2) dos artigos foi denominada "Principais distúrbios na criança indígena". Esta categoria retrata, principalmente os cuidados em relação à diarreia e a presença da infecção tuberculosa nessa população.

A diarreia infantil entre os Kaiowa e Guarani, povos do Estado de Mato Grosso do Sul, pode ser causada pelo coalho virado, uma movimentação brusca das crianças após as refeições, desmame precoce, precárias condições de abastecimento de água e de destino dos dejetos humanos, condições estas relacionadas ao saneamento, acesso aos serviços de saúde, assim como as questões históricas, econômicas e sociais vivenciadas durante décadas de contato com a sociedade não indígena. Os cuidados à criança com diarreia varia desde a procura por especialista tradicional, a utilização de chás ou infusões feitos à base de plantas medicinais, a procura de um pastor para orar pela criança, a realização de massagem no corpo, principalmente barriga e pernas da criança e procura pelo serviço de saúde. ${ }^{13}$ 
O enfermeiro apresenta papel importante na educação em saúde, incentivando a prática do aleitamento materno, orientando quanto aos alimentos disponíveis na região e que possam ser oferecidos à criança a partir dos seis meses de idade, à lavagem dos alimentos consumidos crus e o consumo de água previamente tratada ou tratada no domicílio por fervura ou filtragem.

A terceira categoria denominada "Percepções da morte de criança indígena" foi encontrada em $22 \%$ (2) da amostra de artigos. Esta categoria retrata a compreensão da morte de crianças indígenas, por parte do seu povo e dos profissionais de enfermagem.

Os principais sentimentos que emergem nos profissionais de enfermagem frente à morte de uma criança indígena hospitalizada são impotência, tristeza, culpa por talvez não ter realizado cuidados que deveriam ser prestados e revolta por perceber que a família não deu devida importância ao problema da criança. A falta de conhecimento dos profissionais de enfermagem em relação à língua e à cultura indígena dificulta a assistência, tanto no tratamento, como no momento do óbito e posterior acolhimento da família. Esse aspecto leva à uma preocupação de inserir disciplinas e conteúdos específicos sobre a cultura indígena, nos cursos de formação técnica e superior e valorização de projetos de extensão universitária que levem professores, alunos e profissionais de enfermagem para as comunidades indígenas. ${ }^{14}$

O auxílio ao doente e à família, em um momento em que experimentam grande sofrimento, representa um dos maiores desafios para os profissionais de enfermagem, tornando-se muito mais complexa, quando se trata do atendimento ao indígena, posto que o profissional não tem conhecimento acerca da língua, dos rituais e das crenças desses povos. É fundamental que $\mathrm{o}$ profissional de enfermagem aprimore habilidades para atuar no processo de terminalidade e de luto da família do paciente. Para isso, o mesmo necessita obter conhecimentos e ampliar capacidades e aptidão para encarar a morte, quando se confronta com essa situação, respeitando seus aspectos socioculturais.

A quarta categoria denominada como "Práticas de cuidado com o RN indígena" foi encontrada em 11\% (01) da amostra de artigos. Nesta categoria foram identificadas as principais práticas de cuidado com o RN indígena. 
As práticas de cuidado ao $\mathrm{RN}$ indígena mais frequentes estão relacionadas ao banho, ao aleitamento materno e à limpeza do coto umbilical. O principal cuidado é o banho, com utilização da água morna, pois para as mães, a utilização de água fria poderia ocasionar a morte da criança. Em alguns casos, em âmbito hospitalar, após o parto, os profissionais da saúde não dão banho no $\mathrm{RN}$, apenas o higienizam com pano molhado, tendo como consequência a insatisfação de mães indígenas, que consideram tal situação como um descaso e preconceito, pelo fato de serem indígenas. ${ }^{15}$

Considera-se importante que o conhecimento dos profissionais de enfermagem acerca das práticas de cuidado das famílias indígenas com os $\mathrm{RN}$ pode subsidiar políticas públicas de saúde, como também o planejamento de suas ações, por meio da compreensão dos aspectos culturais que influenciam o processo de cuidado desta população de modo a oferecer atenção diferenciada a este grupo étnico.

\section{CONCLUSÕES}

A busca por evidências apontou que as principais práticas de cuidado ao $\mathrm{RN}$ e criança indígena envolvem banho, aleitamento materno e limpeza do coto umbilical, sendo estes realizados de acordo com o contexto histórico e social dos indígenas, talvez pela falta de orientações por parte da equipe de saúde de como esses cuidados devem ser prestados.

Ainda de acordo com esta RI, os aspectos nutricionais são de maior relevância nos estudos que diz respeito à saúde do RN e criança indígenas. Déficits estaturais foram os mais encontrados, relacionados às condições inadequadas de higiene, alimentação, habitação e dificuldade de acesso aos serviços de saúde. As principais atribuições do enfermeiro frente a esses problemas são atuar como educador em saúde, principalmente durante o pré-natal e puerpério, incentivando a prática do aleitamento materno, orientando quanto aos alimentos que podem ser oferecidos à criança, atuar no processo de terminalidade e de luto da família da criança, promover um diálogo entre enfermeiro e os pais de $\mathrm{RN}$ e crianças indígenas e acompanhar o crescimento e desenvolvimento dos mesmos.

As políticas de saúde voltadas à população indígena buscam o fortalecimento de práticas tradicionais e a 
inclusão destes no serviço de saúde. Sendo assim, é de suma importância o conhecimento do enfermeiro referente às práticas de cuidado realizadas pelas famílias indígenas ao $\mathrm{RN}$ e criança, compreendendo os aspectos culturais que influenciam esse processo de cuidado, de modo a oferecer acolhimento e prestar cuidados de forma humanizada e de qualidade a essa população, respeitando as diferenças a fim de evitar discriminação.

\section{REFERÊNCIAS}

1. Redding GJ, Byrnes CA. Chronic respiratory symptoms and diseases among indigenous children. Pediatr Clin N Am. 2009; 56: 1323-42.

2. King M, Smith A, Gracey $M$. Indigenous health part 2: the underlying causes of the health gap. Lancet. 2009; 374: 76-85.

3. Mondini L, Canó EN, Fagundes U, Lima EES, Rodrigues D, Baruzzi, RG. Condições de nutrição em crianças Kamaiurá - povo indígena do Alto Xingu, Brasil Central. Rev Bras Epidemiol. 2007; 10(1): 39-47.

4. Morais MB, Alves GM, Fagundes-Neto

U. Estado nutricional de crianças índias terenas: evolução do peso e estatura e prevalência atual de anemia. J Pediatr (Rio J). 2005; 81:383-9.

5. Mendes KDS, Silveira RCCP, Galvão CM. Revisão Integrativa: método de pesquisa para a incorporação de evidências na saúde e na Enfermagem. Texto Contexto Enferm. 2008; 17(4): 758-64.

6. Garnelo L, Buchillet D. Taxonomias das doenças entre os índios baniwa (arawak) e desana (tukano oriental) do alto do Rio Nego. Horiz Antropol. 2006; 12(26): 23160.

7. Pompeo DA, Rossi LA, Galvão CM. Revisão integrativa: etapa inicial do processo de validação de diagnóstico de enfermagem. Acta Paul Enferm. 2009; 22(4): 434-8.

8. MOURA-FERREIRA, M. C. Formulário para Coleta de Dados em Pesquisa Bibliográfica. 2008, 01 p.

9. Barreto CTG. Estado nutricional de crianças indígenas Guarani no sudeste do Brasil. Rio de Janeiro. Dissertação [Mestrado em Saúde Pública] - Escola Nacional de Saúde Pública Sérgio Arouca; 2011.

10. Menegolla IA, Drachler ML, Rodrigues IH, Schwingel LR, Scapinello E, Pedroso MB, Leite JCC. Estado nutricional e fatores associados à estatura de crianças da Terra Indígena Guarita, Sul do Brasil. Cad. Saúde Pública. 2006; 22(2):395-406.

11. Ribas DLB, Sganzerla A, Zorzatto JR, Philippi ST. Nutrição e saúde infantil em uma comunidade indígena Teréna, Mato Grosso do Sul, Brasil. Cad Saúde Pública. 2001; 17(2): 323-31.

12. Ferreira AA. Estado nutricional e fatores associados ao crescimento de crianças indígenas Xavante, Mato Grosso. Rio de Janeiro. Dissertação [Mestrado em Saúde Pública] - Escola Nacional de Saúde Pública Sérgio Arouca; 2009.

13. Pícoli RP, Adorno RCF. Cuidado à saúde de crianças Kaiowá e Guarani: notas de observação de campo. Rev Bras Crescimento Desenvolv Hum. 2008;18(1):35-45.

14. Oliveira PR, Shirmbeck TM, Lunardia RR. Vivências de uma equipe de Enfermagem com a morte de criança indígena hospitalizada. Texto Contexto Enferm Out-Dez. 2013; 22(4): 1072-80. 
15. Rissardo LK, Moliterno ACM, Borghi AC, Carreira L. Práticas de cuidado ao recém-nascido: percepção de famílias Kaingang. Cienc Cuid Saúde. 2011; 10(4): 634-41.

Recebido em 31/10/2015

Aprovado em 08/06/2016

Publicado em 29/12/2016 